\title{
9-(4-Bromophenyl)-9-fluorenyl as a Safety-Catch Nitrogen Protecting Group
}

Simon Surprenant, and William D. Lubell*

Département de chimie, Université de Montréal

C.P. 6128, Succursale Centre Ville, Montréal, Québec, Canada H3C 3J7

lubell@chimie.umontreal.ca

\section{Supporting Information}

Table of contents

General Experimental Section

Spectral data for $\mathbf{6}$

Spectral data for $\mathbf{1 2}$

Spectral data for $\mathbf{8}$

Spectral data for $\mathbf{1 0}$

Spectral data for $\mathbf{1 3}$

S4

Proton NMR spectrum for $\mathbf{2}$

Carbon NMR spectrum for $\mathbf{2}$

Proton NMR spectrum for $\mathbf{3}$ 
Carbon NMR spectrum for $\mathbf{6}$

Proton NMR spectrum for $\mathbf{1 1}$

Carbon NMR spectrum for $\mathbf{1 1}$

Proton NMR spectrum for $\mathbf{1 2}$

Carbon NMR spectrum for 12

Proton NMR spectrum for $\mathbf{1 3}$

Carbon NMR spectrum for $\mathbf{1 3}$

Proton NMR spectrum for $\mathbf{1 4}$

Carbon NMR spectrum for $\mathbf{1 4}$

Proton NMR spectrum for 15

Carbon NMR spectrum for 15

Proton NMR spectrum for 16

Carbon NMR spectrum for $\mathbf{1 6}$

HPLC trace of competitive cleavage experiment with compound 2 
General Experimental Section. Unless otherwise noted, all reactions were performed under argon atmosphere and distilled solvents were transferred by syringe. Anhydrous $\mathrm{CH}_{2} \mathrm{Cl}_{2}$ was obtained from a solvent filtration system. Final reaction mixture solutions were dried over anhydrous $\mathrm{MgSO}_{4}$, filtered and rotary-evaporated under reduced pressure. Unless otherwise noted, ${ }^{1} \mathrm{H}$ NMR (300/400 MHz) and ${ }^{13} \mathrm{C}$ NMR $(75 / 100$ $\mathrm{MHz})$ spectra were recorded in $\mathrm{CDCl}_{3}$. Chemical shifts are reported in ppm ( $\delta$ units) downfield from internal tetramethylsilane $\left((\mathrm{CH})_{4} \mathrm{Si}\right)$, residual $\mathrm{CHCl}_{3}(\delta 7.27$ and $77.2 \mathrm{ppm}), \mathrm{C}_{6} \mathrm{D}_{6}(\delta 7.16$ and $128.1 \mathrm{ppm}), \mathrm{CD}_{3} \mathrm{OD}(3.31$ and $49.0 \mathrm{ppm})$ or $\mathrm{D}_{2} \mathrm{O}$ (4.79). Aromatic carbons of compounds having a PhF group or a derivative are reported as seen on spectra. Analytical thin-layer chromatography (TLC) was performed by using glass-backed silica gel plates coated with a $0.2 \mathrm{~mm}$ thickness of silica gel. Compounds were purified by column chromatography using $230-400$ mesh silica gel.

(2S)-N-(MPF)Alanine tert-Butyl Ester (6) Chromatography of the product from 5 (230 mg, 0.50 mmol) using $20 \%$ EtOAc in hexanes as eluant gave 6 (186 mg, $79 \%$ yield) as a white foam: mp 63-65두 $[\alpha]^{20}{ }_{\mathrm{D}}-115.7^{\circ}\left(c\right.$ 1.1, $\left.\mathrm{CH}_{3} \mathrm{OH}\right) ;{ }^{1} \mathrm{H} \mathrm{NMR} \delta 7.69(\mathrm{dd}, J=7.6 \mathrm{~Hz}, 1.1 \mathrm{~Hz}, 2 \mathrm{H}), 7.36-$ $7.23(\mathrm{~m}, 8 \mathrm{H}), 6.77$ (d, $J=8.9 \mathrm{~Hz}, 2 \mathrm{H}), 3.83(\mathrm{t}, J=4.8 \mathrm{~Hz}, 4 \mathrm{H}), 3.11(\mathrm{t}, J=4.8 \mathrm{~Hz}, 4 \mathrm{H}), 2.78(\mathrm{q}, J=7.1 \mathrm{~Hz}, 1 \mathrm{H}), 1.23(\mathrm{~s}, 9 \mathrm{H}), 1.12(\mathrm{~d}, J=7.1 \mathrm{~Hz}$ 3H); ${ }^{13}$ C NMR $\delta 176.4,150.5,141.0,140.5,128.4,128.2,128.1,127.5,126.3,125.6,120.3,120.1,115.6,80.7,73.1,67.3,52.4,49.6,28.3,22.6$; HRMS calcd for $\mathrm{C}_{30} \mathrm{H}_{34} \mathrm{~N}_{2} \mathrm{O}_{3} \mathrm{Na}[\mathrm{M}+\mathrm{Na}]^{+}$493.2461, found 493.2459.

$\boldsymbol{N}$-(MPF)-Alaninyl-( $\omega$-Boc)lysine tert-butyl ester (12) Chromatography of the product from 11 (300 mg, $0.43 \mathrm{mmol})$ using $50 \%$ EtOAc in hexanes as eluant gave 12 (235 mg, $78 \%$ yield) as a yellowish oil; $[\alpha]^{20}{ }_{\mathrm{D}} 12.5^{\circ}\left(c\right.$ 1.0, $\left.\mathrm{CH}_{3} \mathrm{OH}\right) ;{ }^{1} \mathrm{H} \mathrm{NMR}\left(\mathrm{C}_{6} \mathrm{D}_{6}\right) \delta 7.88(\mathrm{~m}, 2 \mathrm{H}), 7.77(\mathrm{~d}, J=8.8$ Hz, 2H), 7.61 (m, 3H), 7.37-7.18 (m, 5H), $6.72(\mathrm{~d}, J=8.9 \mathrm{~Hz}, 2 \mathrm{H}), 4.68(\mathrm{~m}, 1 \mathrm{H}), 4.47(\mathrm{~m}, 1 \mathrm{H}), 3.60(\mathrm{t}, J=4.8 \mathrm{~Hz}, 4 \mathrm{H}), 3.11(\mathrm{~m}, 2 \mathrm{H}), 2.84(\mathrm{~m}$, $1 \mathrm{H}), 2.77(\mathrm{t}, J=4.8 \mathrm{~Hz}, 4 \mathrm{H}), 1.89(\mathrm{~m}, 1 \mathrm{H}), 1.66(\mathrm{~m}, 1 \mathrm{H}), 1.55(\mathrm{~s}, 9 \mathrm{H}), 1.47(\mathrm{~s}, 9 \mathrm{H}), 1.35(\mathrm{~m}, 4 \mathrm{H}), 1.09(\mathrm{~d}, J=7.1 \mathrm{~Hz}, 3 \mathrm{H}) ;{ }^{13} \mathrm{C} \mathrm{NMR}\left(\mathrm{C}_{6} \mathrm{D}_{6}\right) \delta$ 175.2, 172.7, 151.2, 150.7, 149.4, 141.8, 140.5, 135.9, 128.8, 128.7, 127.6, 127.4, 126.8, 125.1, 120.5, 120.4, 116.0, 81.6, 78.4, 73.6, 67.0, 53.3, 52.3, 49.4, 40.7, 33.5, 28.7, 28.2, 28.1, 22.7, 22.2; HRMS calcd for $\mathrm{C}_{41} \mathrm{H}_{54} \mathrm{~N}_{4} \mathrm{O}_{6} \mathrm{Na}[\mathrm{M}+\mathrm{Na}]^{+}$721.39356, found 721.39346 . 
(2S)- $N$-(Boc)Alanine Methyl Ester (8) Chromatography of the product from 2 (50 mg, 0.12 mmol) using $10 \%$ EtOAc in hexanes as eluant gave 7 as a white solid (19.0 mg, $79 \%$ yield): $\mathrm{mp} 31-33^{\circ} \mathrm{C}$, lit. ${ }^{21} \mathrm{mp} 30^{\circ} \mathrm{C}$; $[\alpha]^{20}{ }_{\mathrm{D}}-45.1^{\circ}\left(\mathrm{c} 1.0, \mathrm{CH}_{3} \mathrm{OH}\right), \mathrm{lit}^{21}[\alpha]^{20}{ }_{\mathrm{D}}-44^{\circ}(\mathrm{c} 1.0, \mathrm{CH} \mathrm{OH}) . \mathrm{The}^{2}$ ${ }^{1} \mathrm{H}$ and ${ }^{13} \mathrm{C}$ NMR spectral data were consistent with the literature. ${ }^{21}$

(2S)- $\boldsymbol{N}$-(Boc)Alanine tert-Butyl Ester (10) Chromatography of the product from 6 (50 mg, $0.11 \mathrm{mmol}$ ) using $10 \%$ EtOAc in hexanes as eluant gave 10 as a clear oil (22.8 mg, $84 \%$ yield): $[\alpha]^{20}{ }_{\mathrm{D}}-36.7^{\circ}\left(c\right.$ 1.0, $\left.\mathrm{CH}_{3} \mathrm{OH}\right)$, lit. ${ }^{22}[\alpha]_{\mathrm{D}}^{20}-38.1^{\circ}\left(c 1.0, \mathrm{CH}_{3} \mathrm{OH}\right)$. The ${ }^{1} \mathrm{H}$ and ${ }^{13} \mathrm{C}$ NMR spectral data were consistent with the literature. ${ }^{23}$

$N$-(Methyl carbamoyl)alaninyl- $\omega$-(Boc)lysine tert-butyl ester (13) Chromatography of the product from 12 (20 mg, 0.029 mmol) using 60 \% EtOAc in hexanes as eluant gave 13 as a yellowish oil (10.1 mg, $82 \%$ yield): mp $105-106^{\circ} \mathrm{C}[\alpha]^{20}{ }_{\mathrm{D}}-11.7^{\circ}\left(\mathrm{c} 1.1, \mathrm{CHCl}_{3}\right)$; ${ }^{1} \mathrm{H} \mathrm{NMR}\left(\mathrm{C}_{6} \mathrm{D}_{6}\right) \delta 7.40$ (d, $J=7.5 \mathrm{~Hz}, 1 \mathrm{H}), 6.33$ (d, $J=7.9 \mathrm{~Hz}, 1 \mathrm{H}), 5.33$ (bs, 1H), $4.76(\mathrm{~m}, 1 \mathrm{H}), 4.44(\mathrm{t}, J=6.9 \mathrm{~Hz}, 1 \mathrm{H}), 3.58(\mathrm{~s}, 3 \mathrm{H}), 3.21(\mathrm{~m}, 1 \mathrm{H}), 3.13(\mathrm{~m}, 1 \mathrm{H}), 1.89$ (m, 1H), $1.71(\mathrm{~m}, 1 \mathrm{H}), 1.43(\mathrm{~s}, 9 \mathrm{H}), 1.47$ (d, $J=7.1 \mathrm{~Hz}, 3 \mathrm{H}) 1.43(\mathrm{~s}, 9 \mathrm{H}), 1.45-1.40(\mathrm{~m}, 4 \mathrm{H}) ;{ }^{13} \mathrm{C}$ NMR $\left(\mathrm{C}_{6} \mathrm{D}_{6}\right) \delta 172.6,171.1156 .7,155.6,80.7$, 77.9, 52.6, 51.4, 50.1, 39.8, 31.6, 29.2, 28.0, 27.3, 22.3, 18.5; HRMS calcd for $\mathrm{C}_{20} \mathrm{H}_{37} \mathrm{~N}_{3} \mathrm{O}_{7} \mathrm{Na}[\mathrm{M}+\mathrm{Na}]^{+}$454.25237, found 454.25195. 


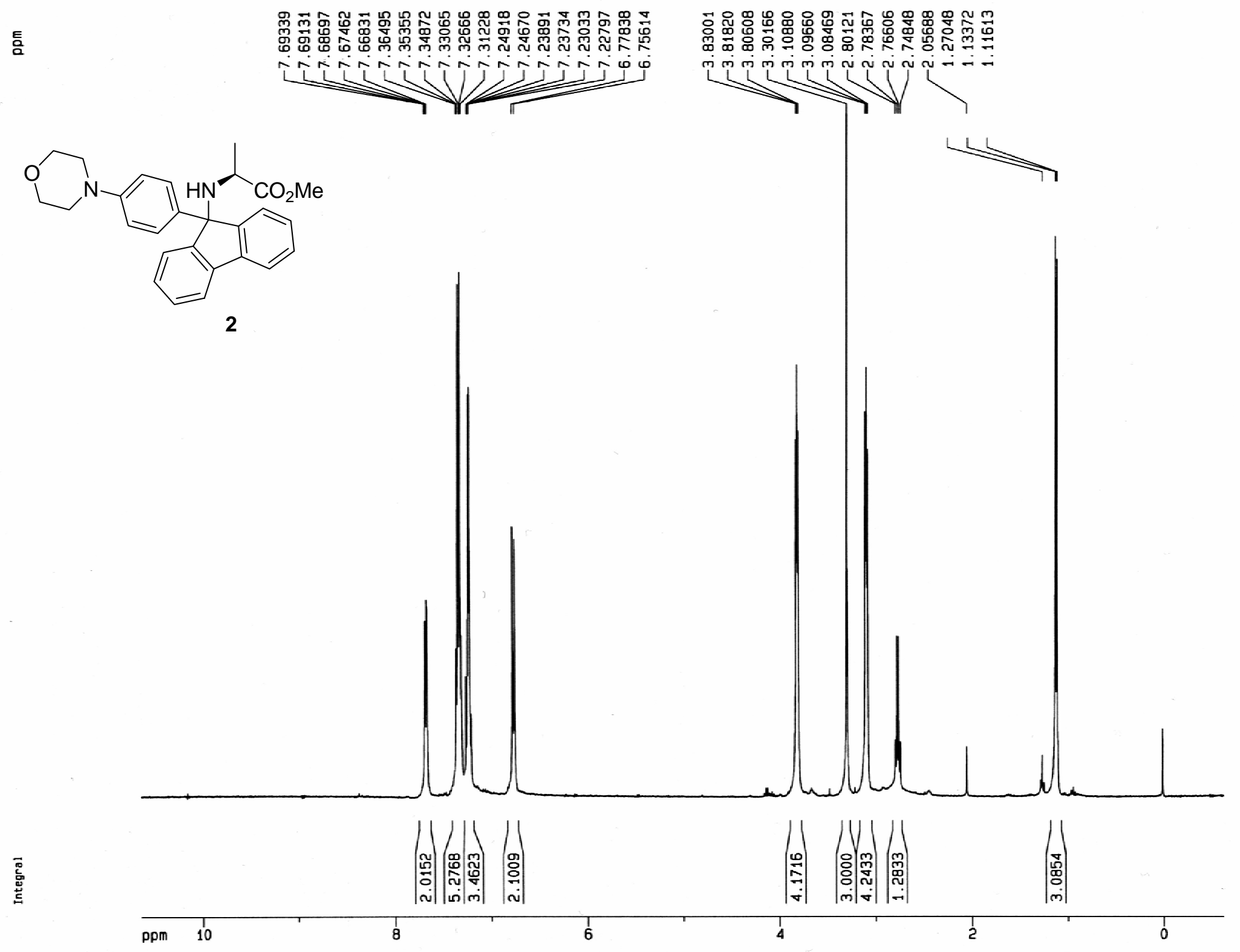



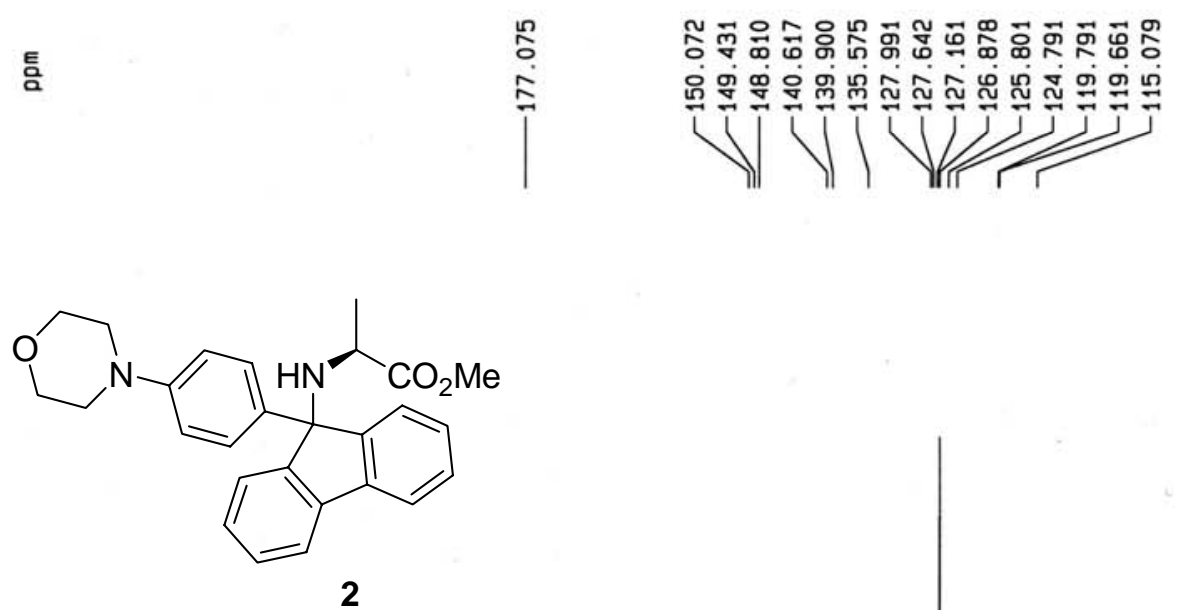

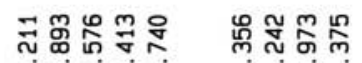

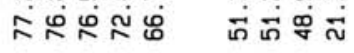

2

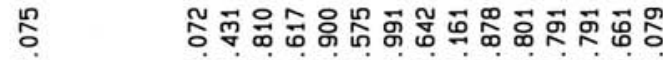

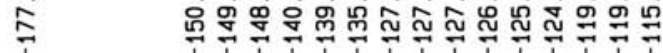

YI Y

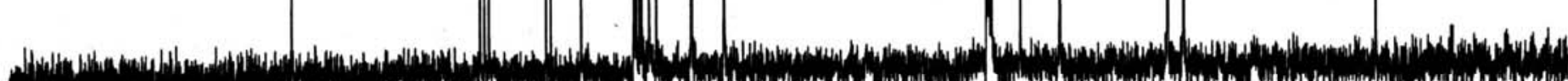

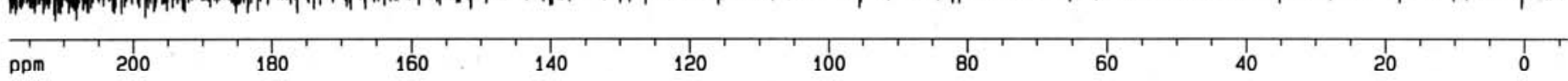


言

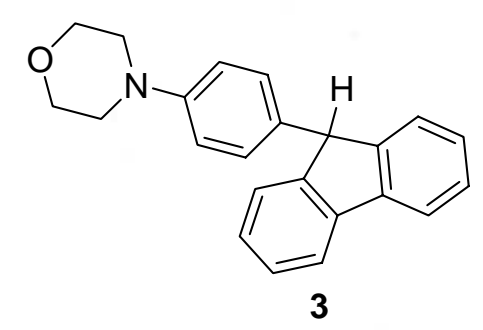

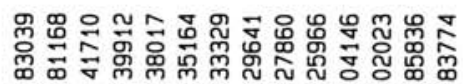

îi

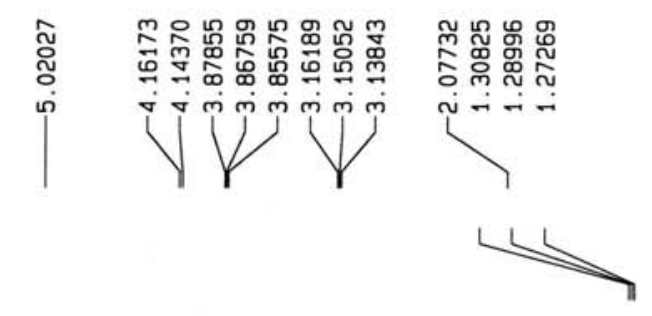

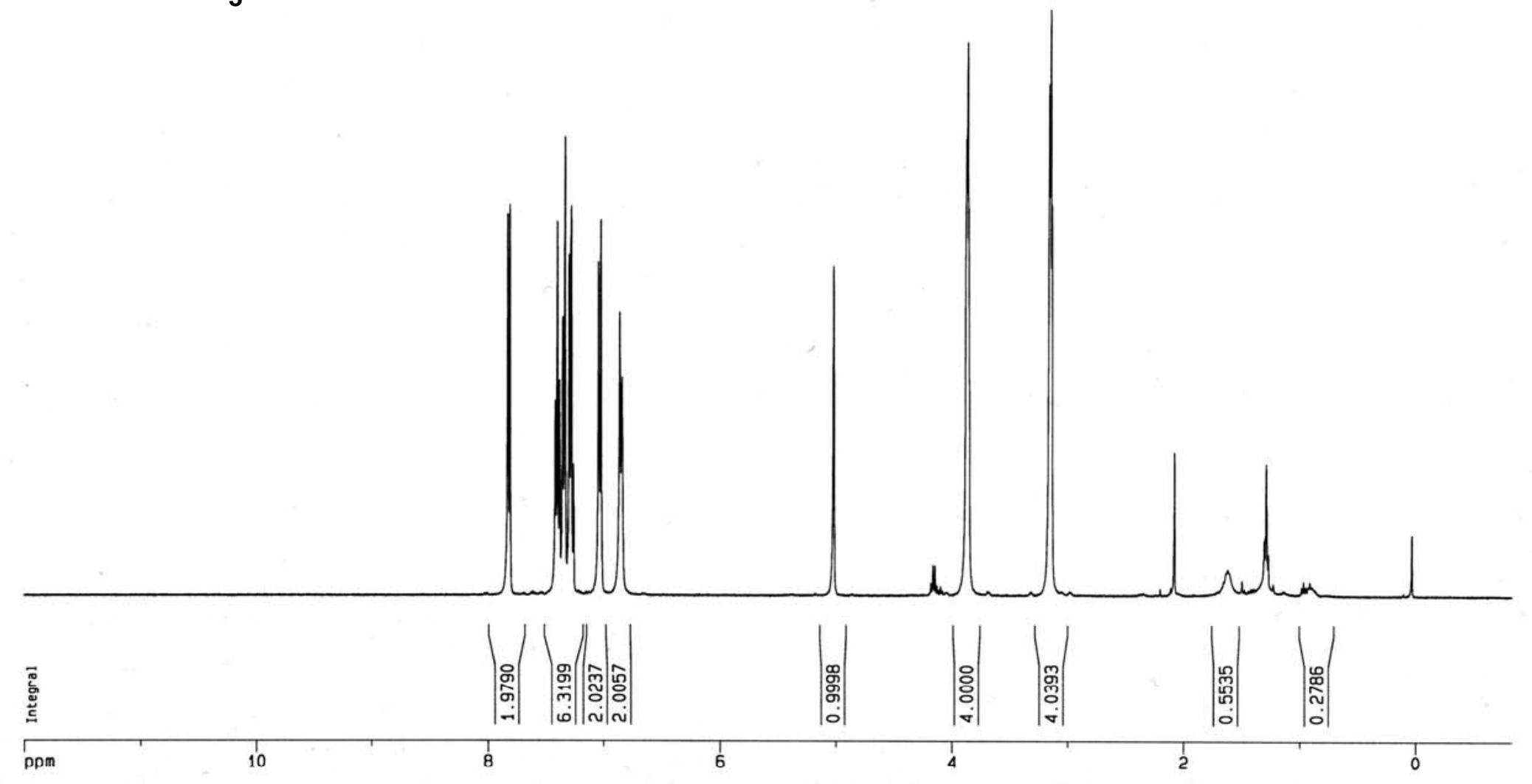




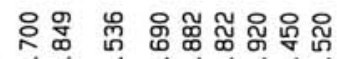

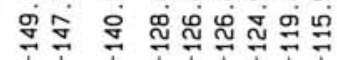

iा एj

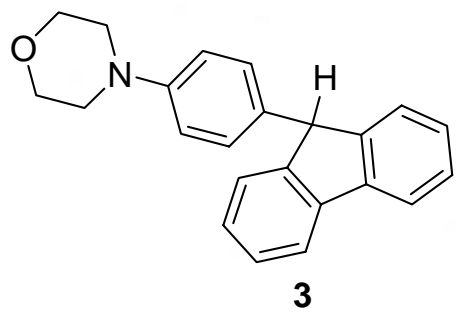

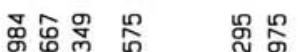

ம்

กิ

V

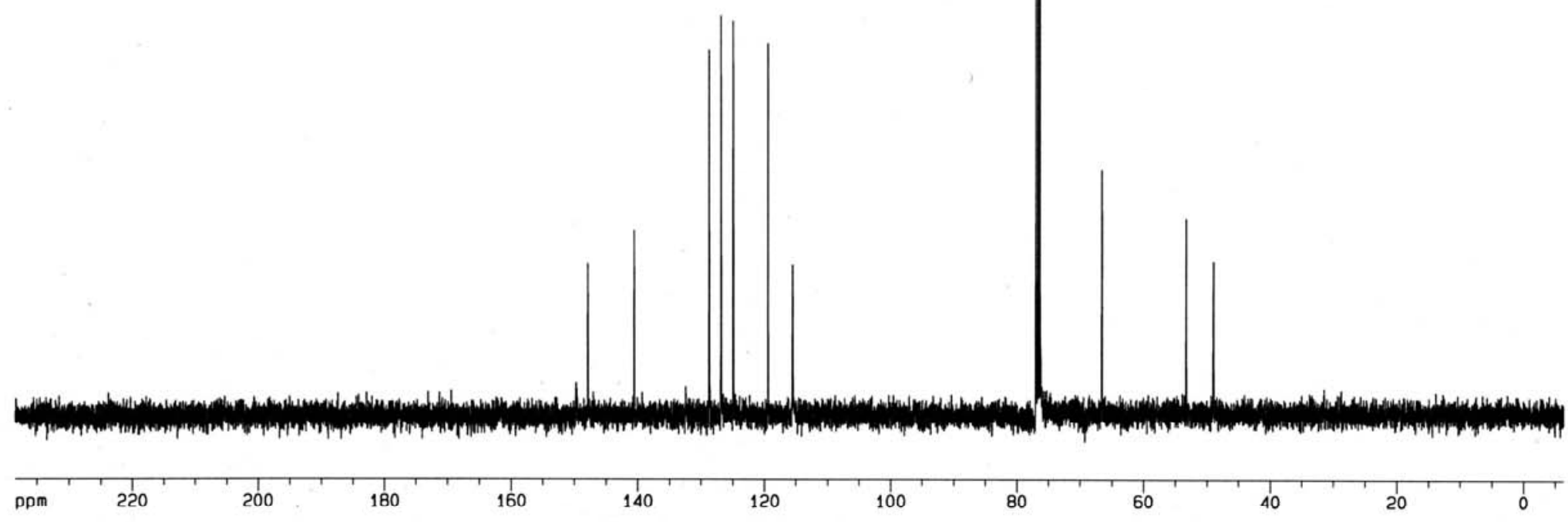




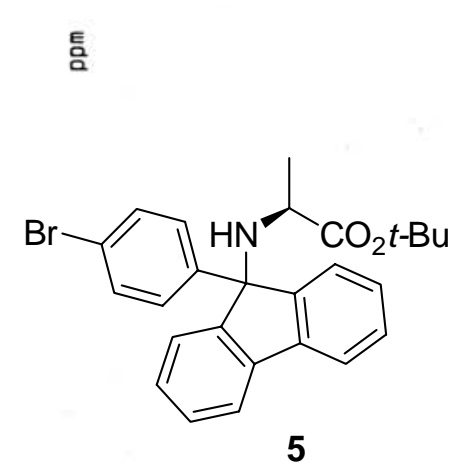

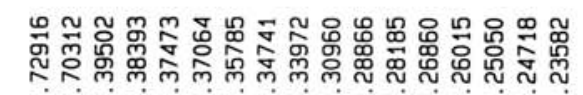

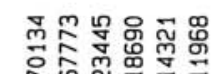

vint

iiiiijijij

\ั
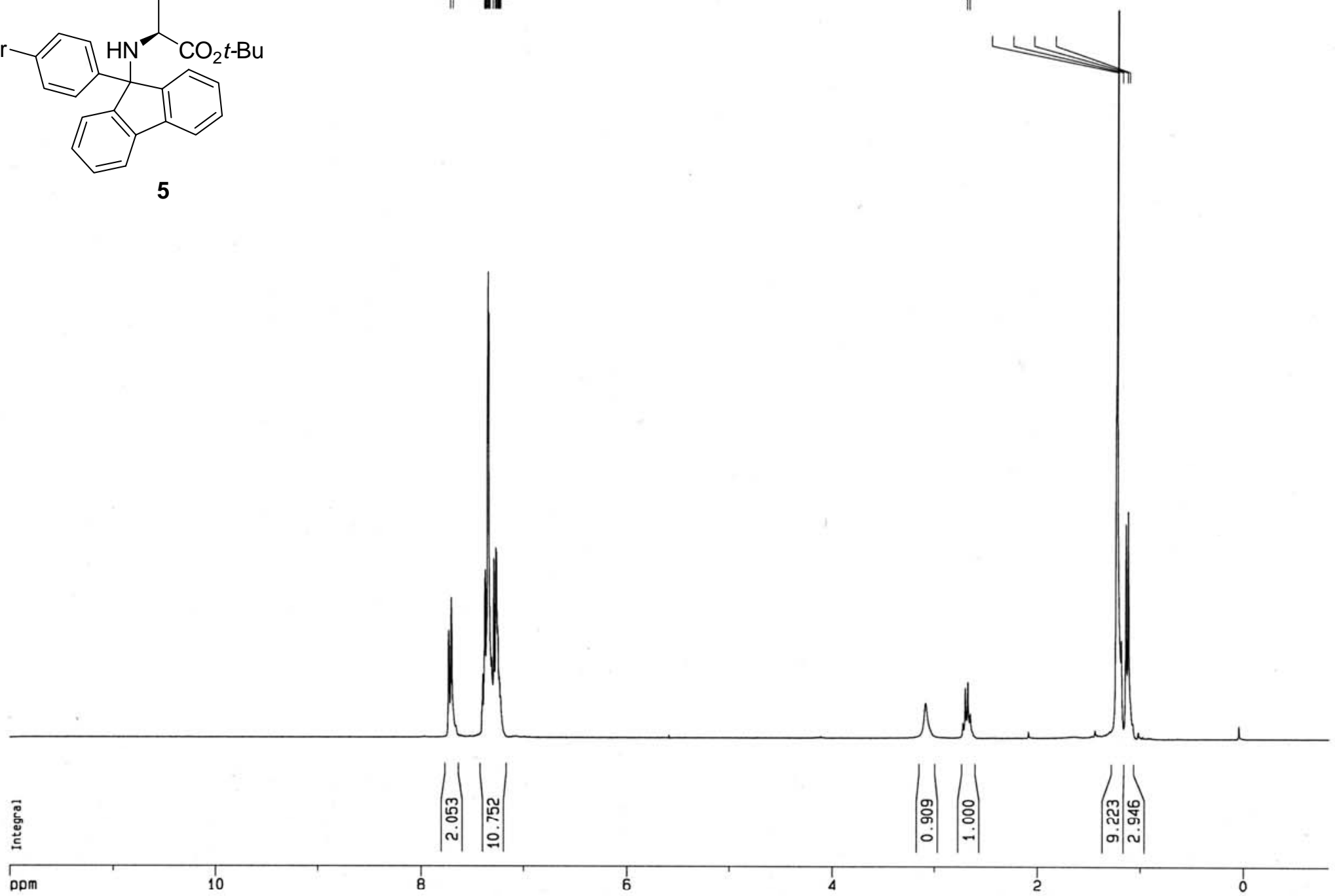


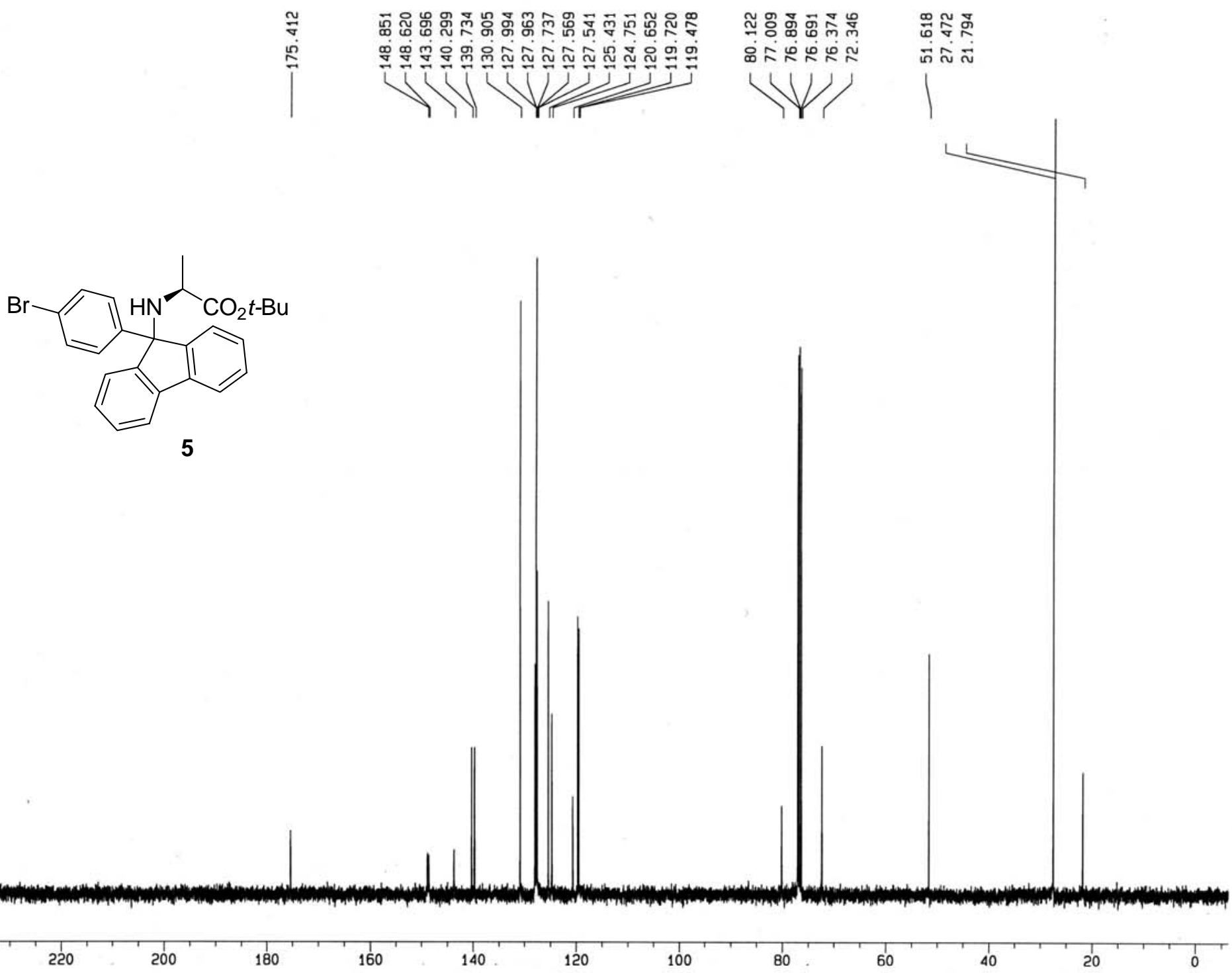



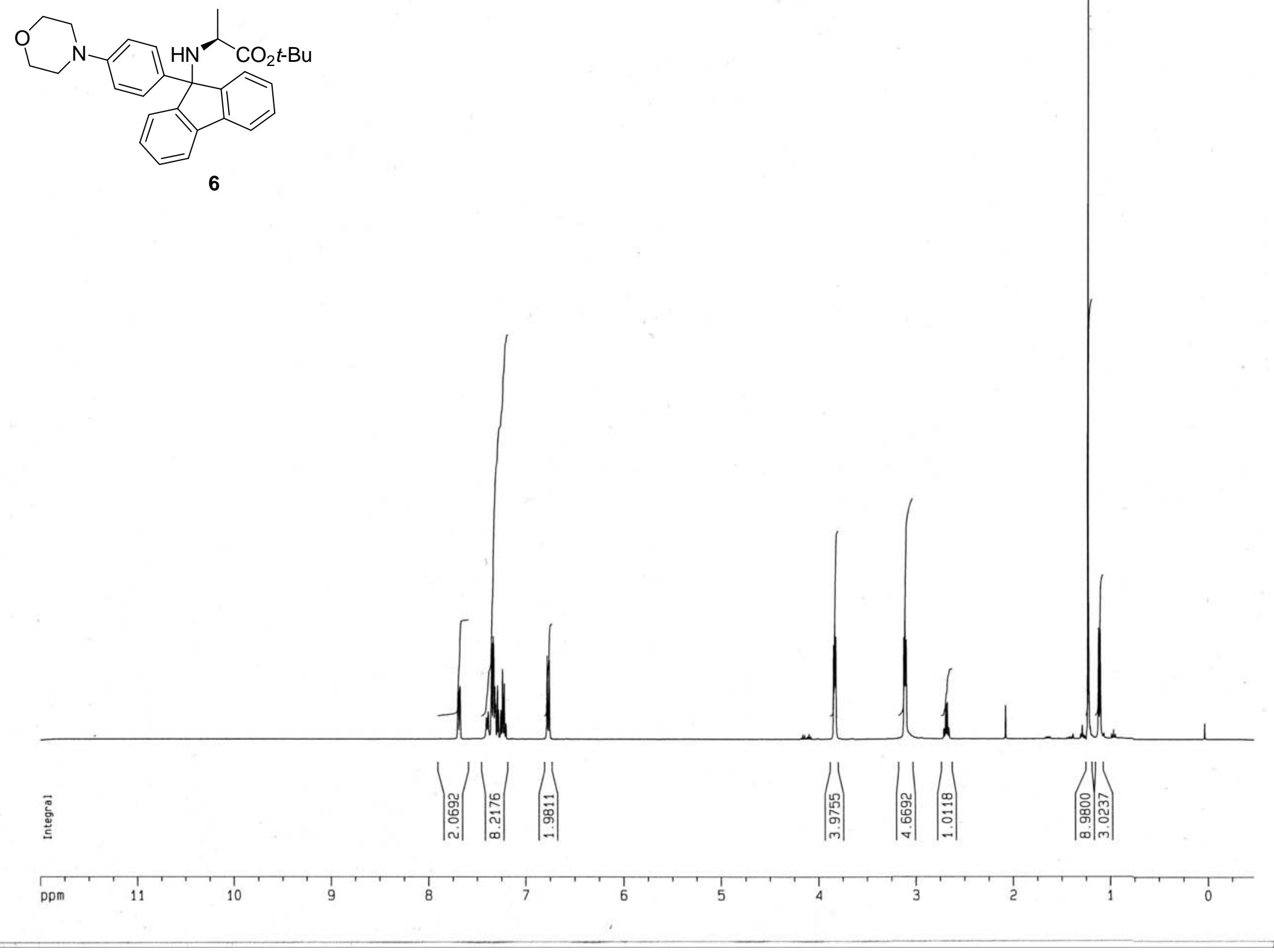

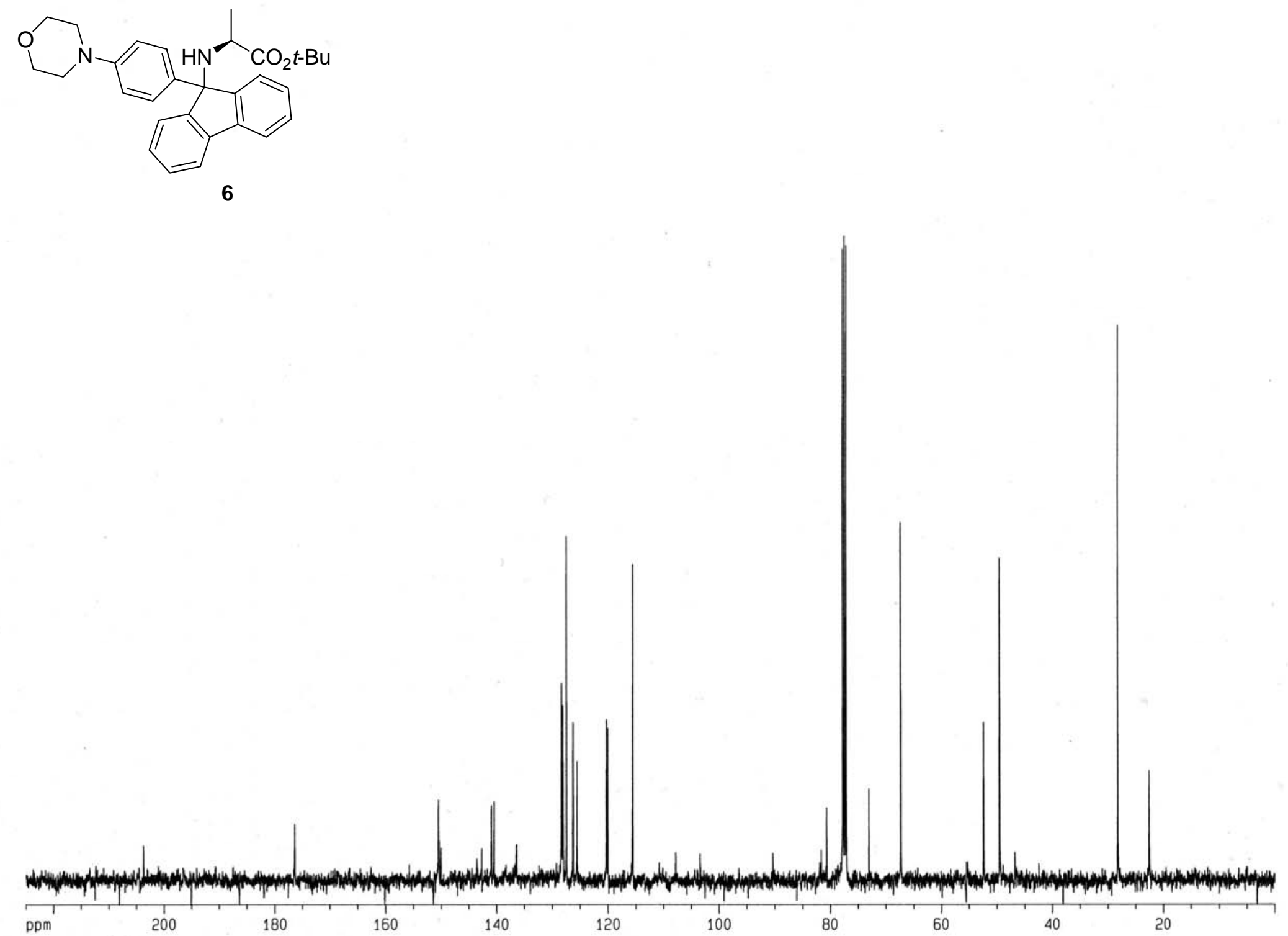
言

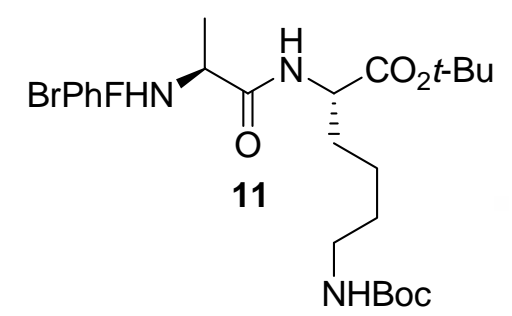

NHBoc

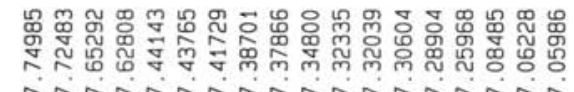

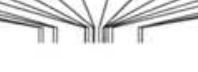

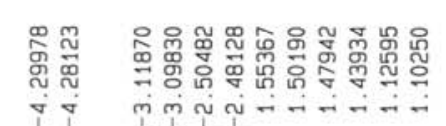

$\gamma$ in

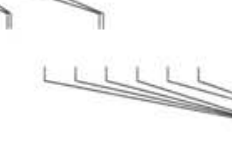

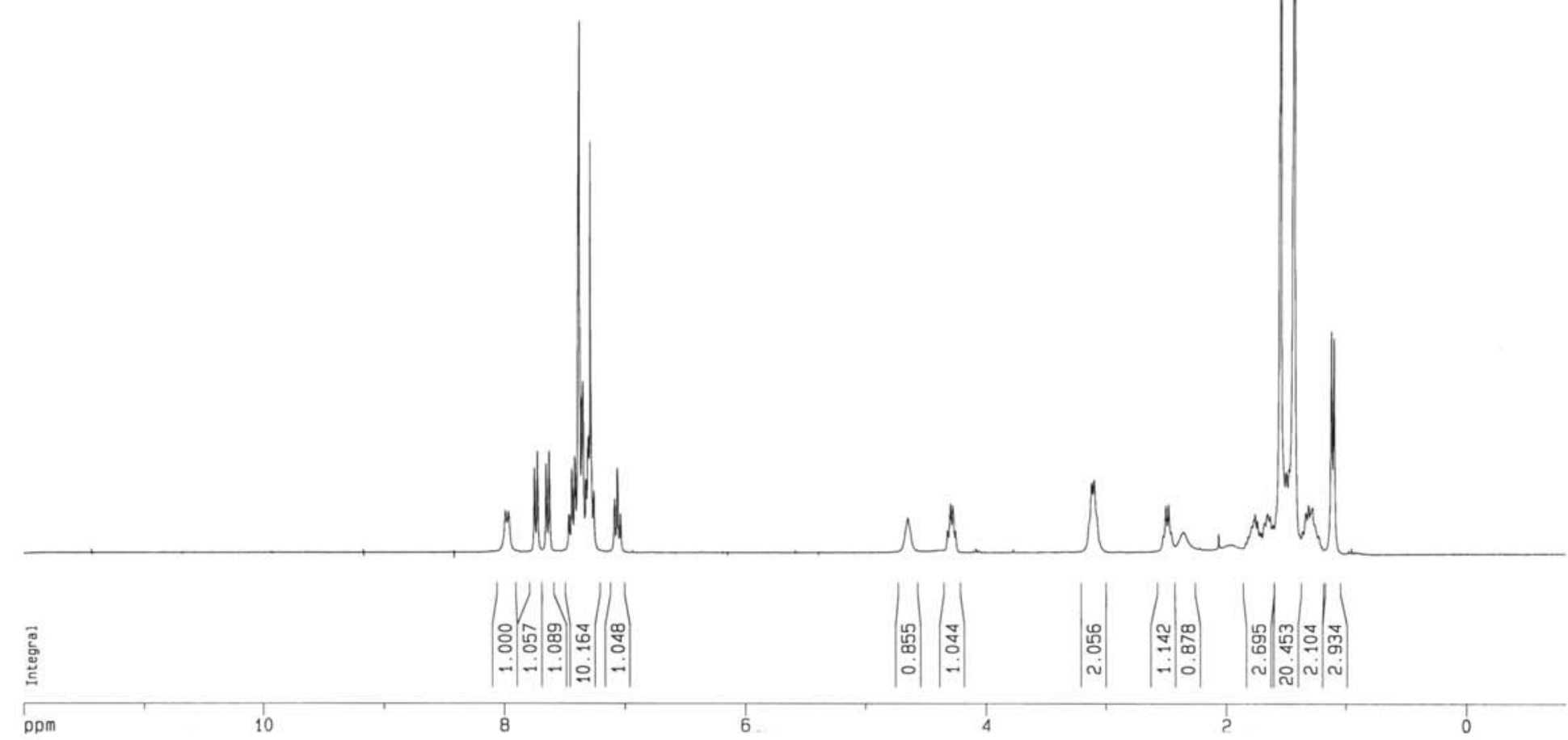




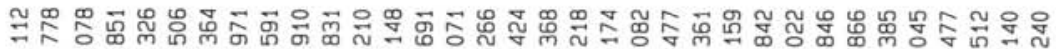

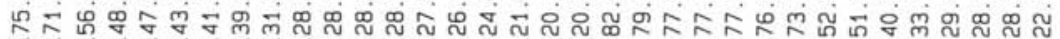
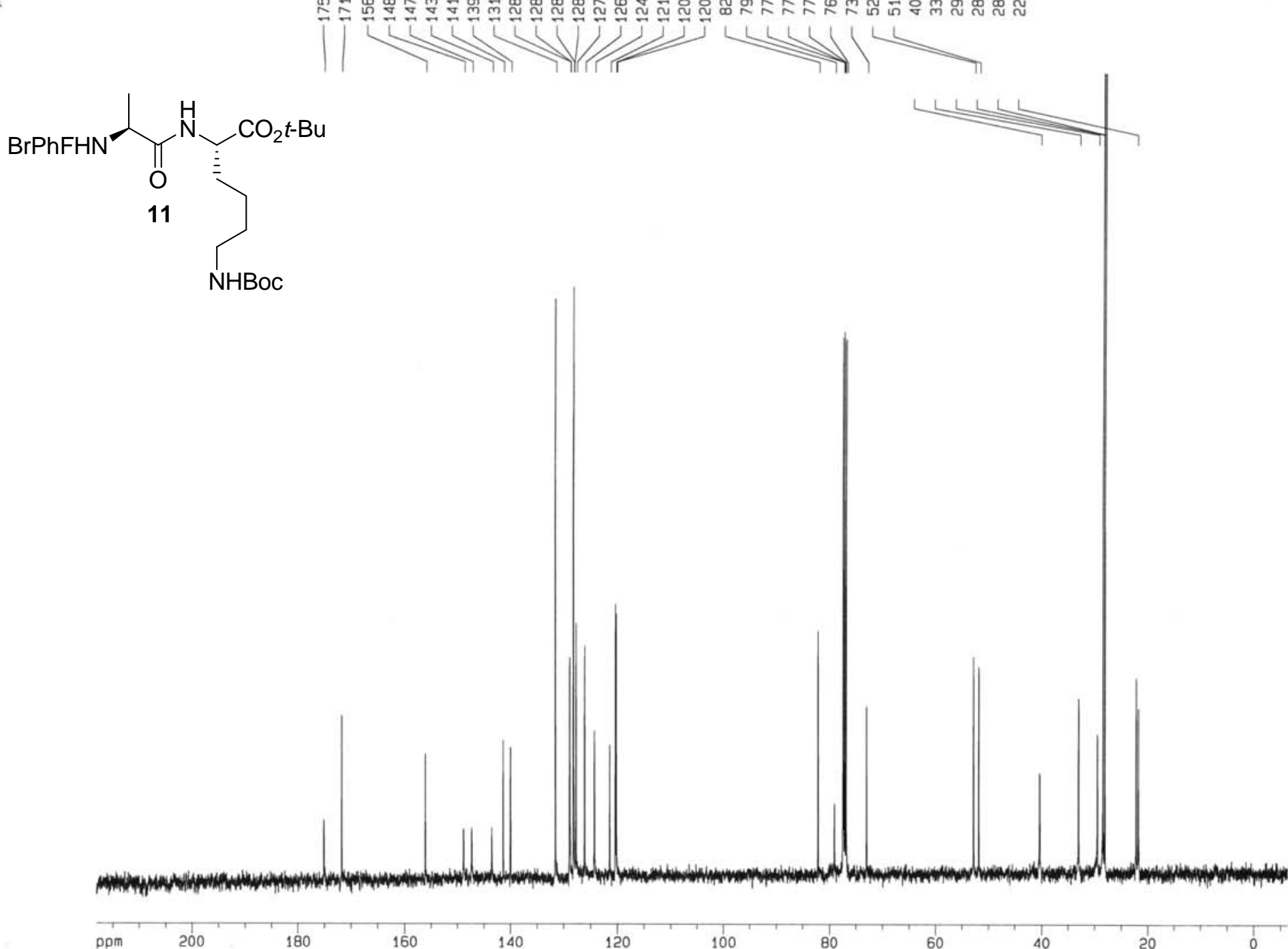


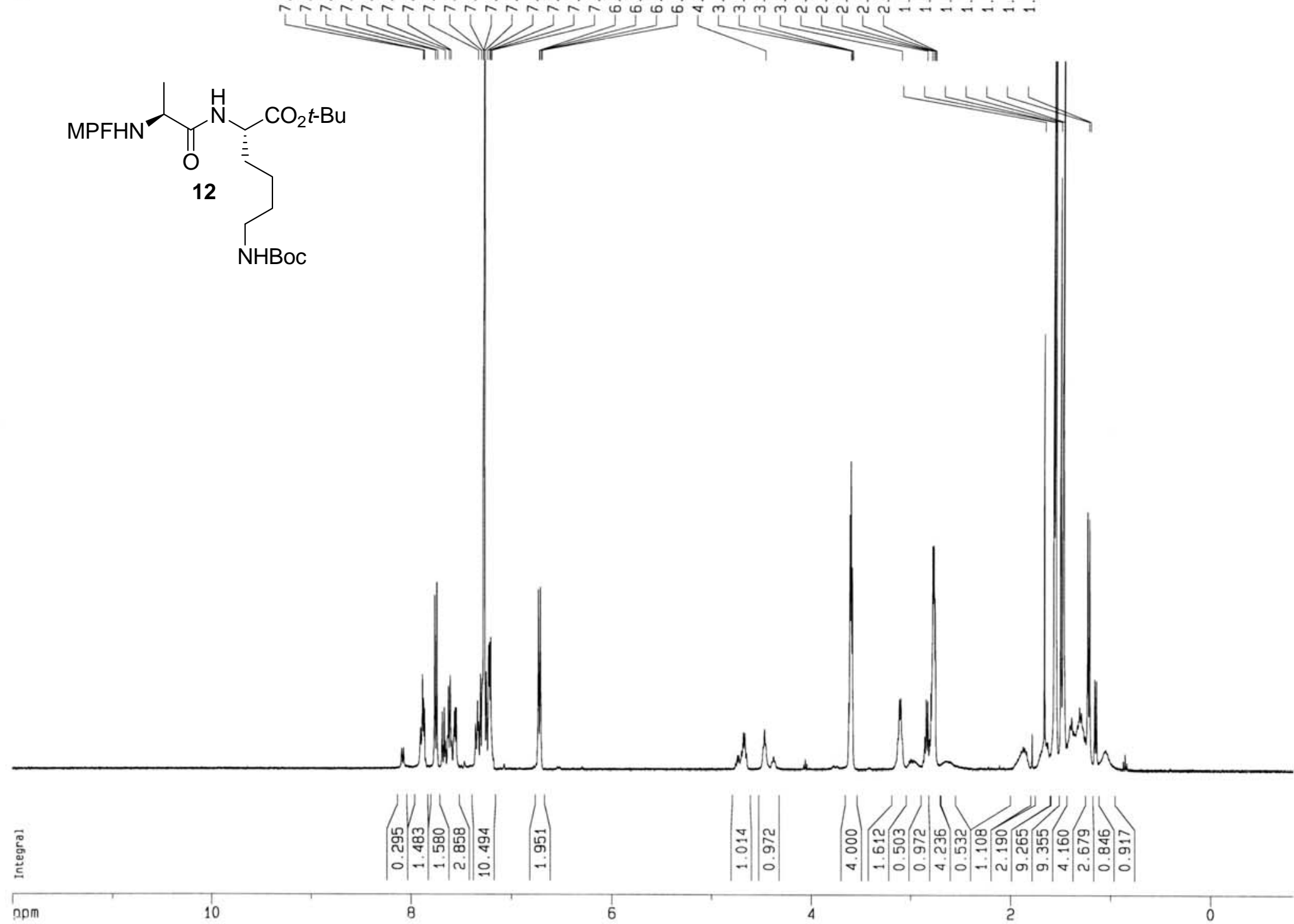




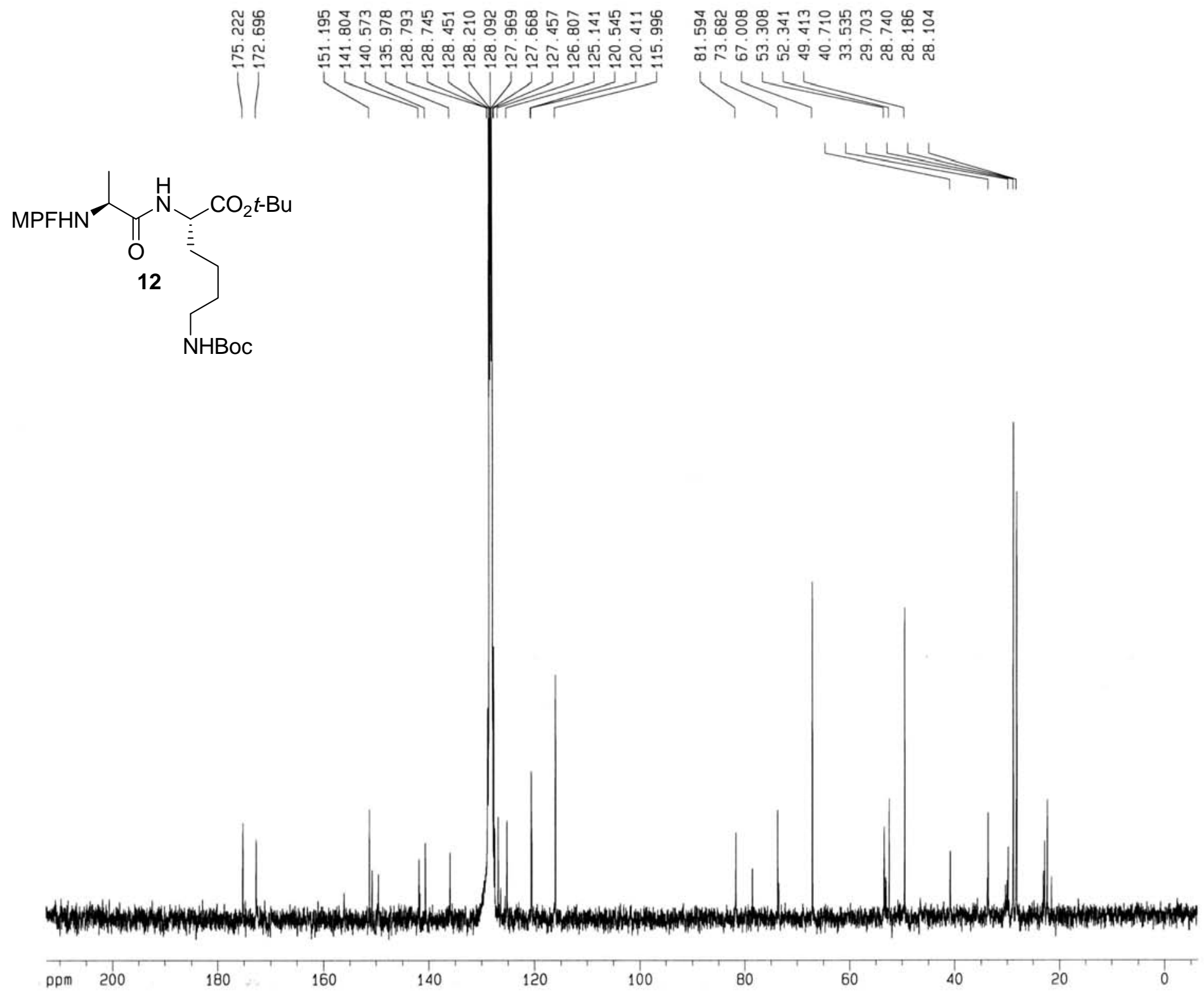




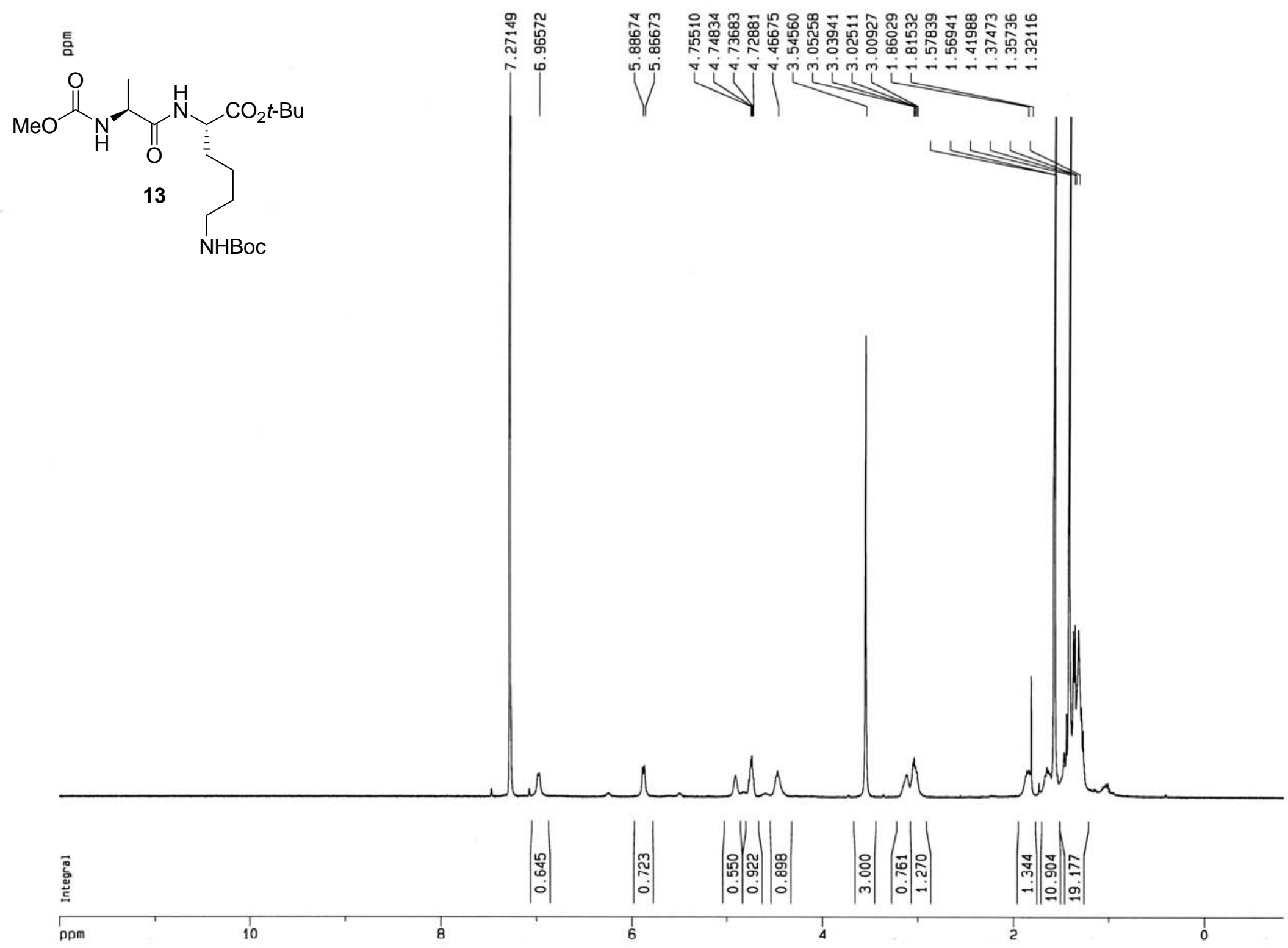




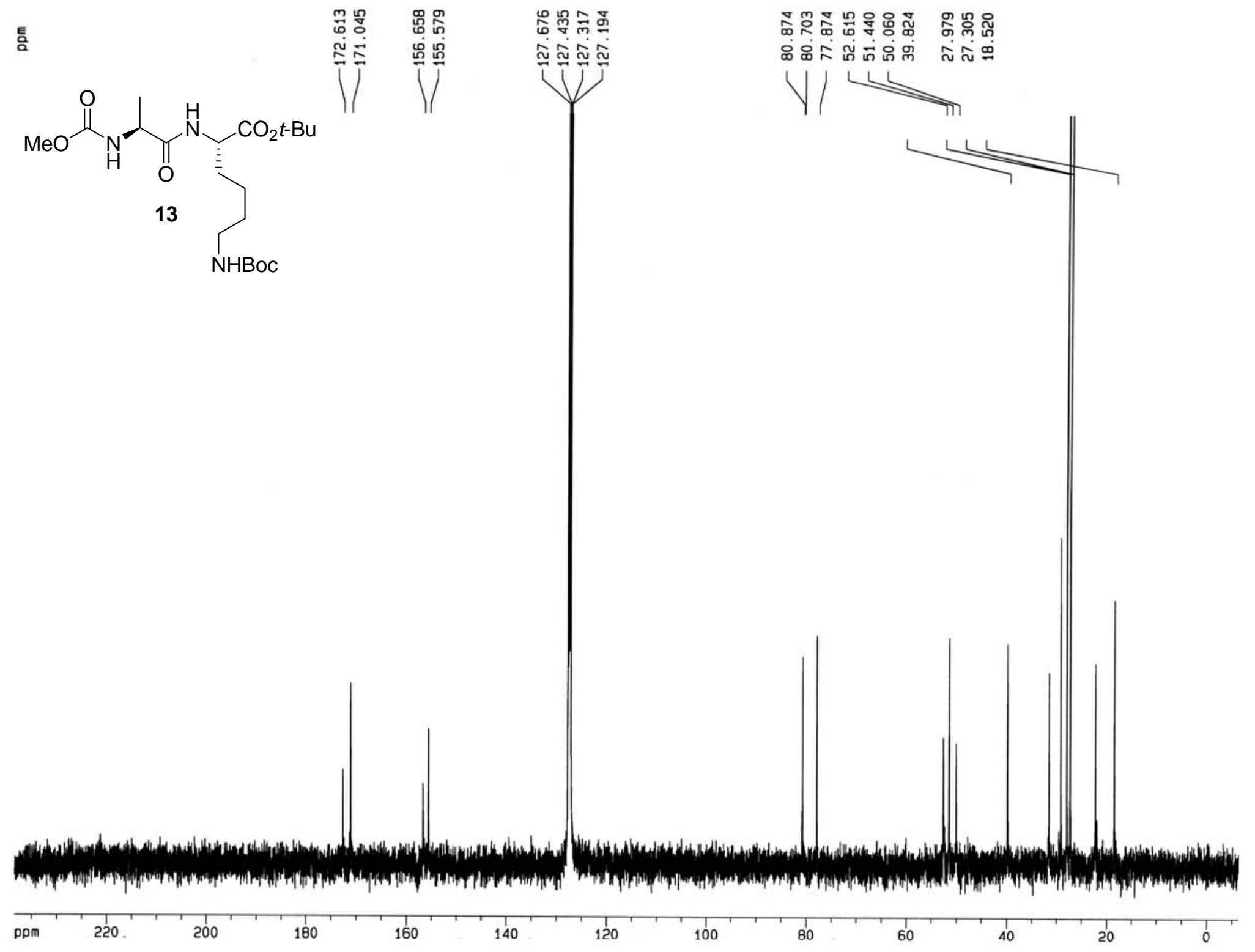




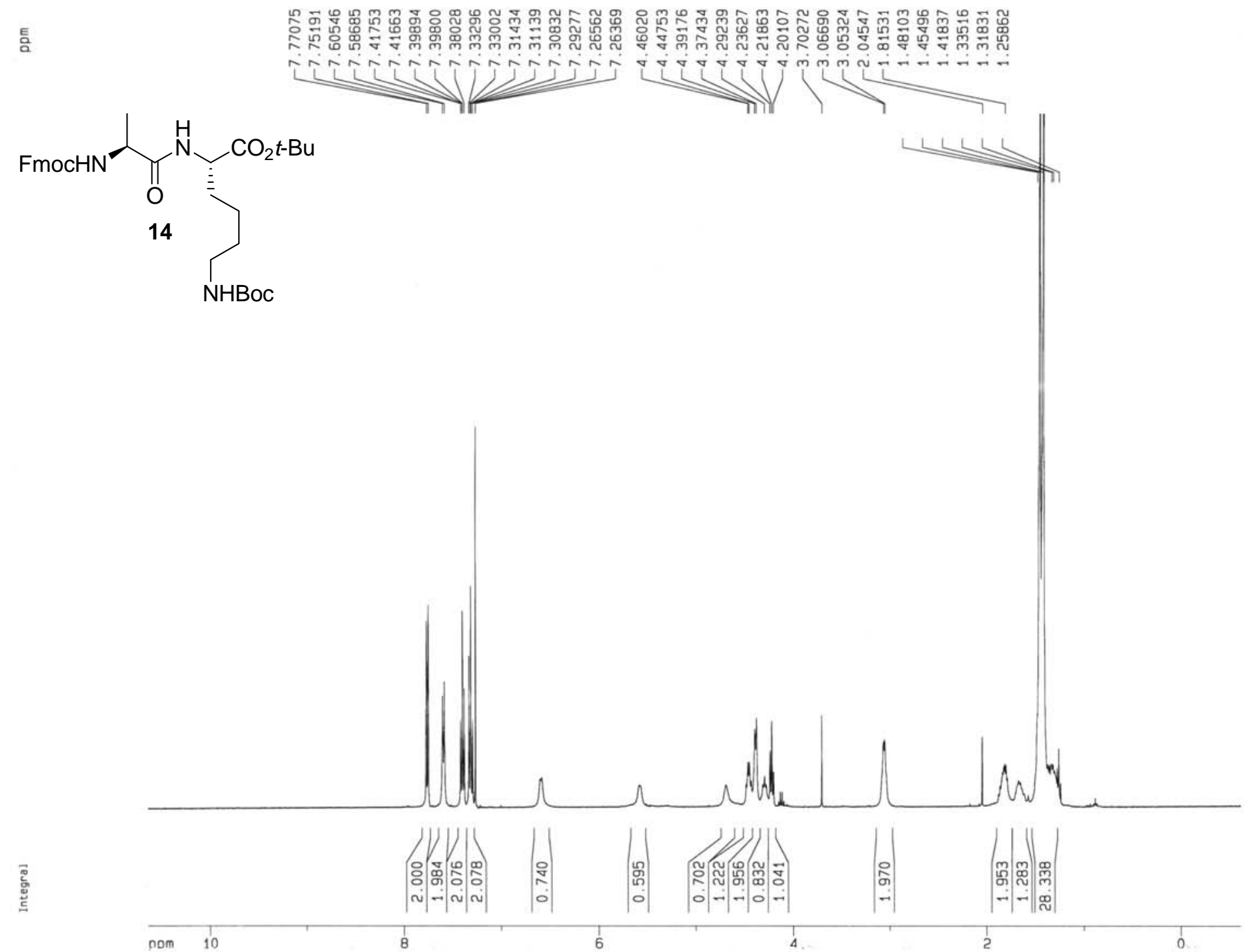




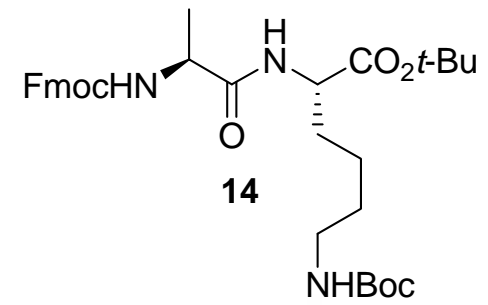

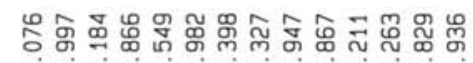

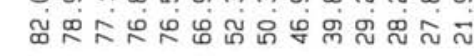

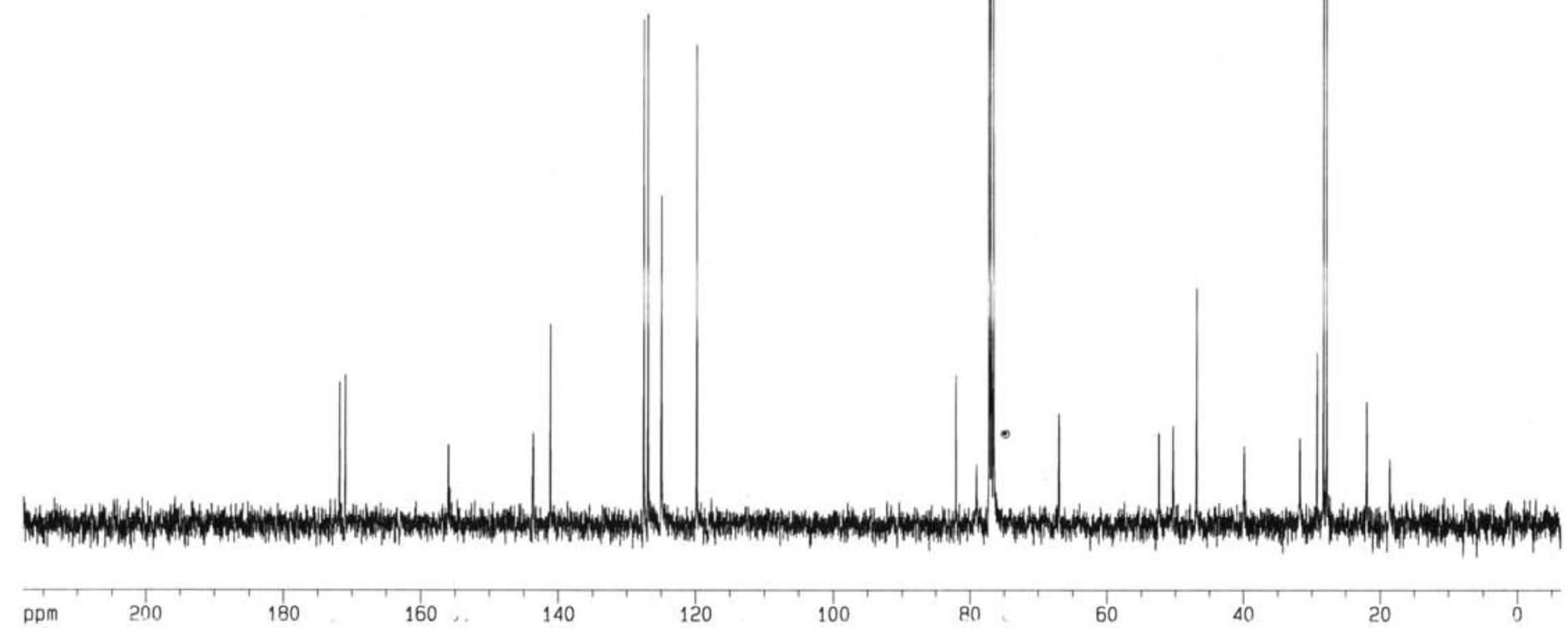




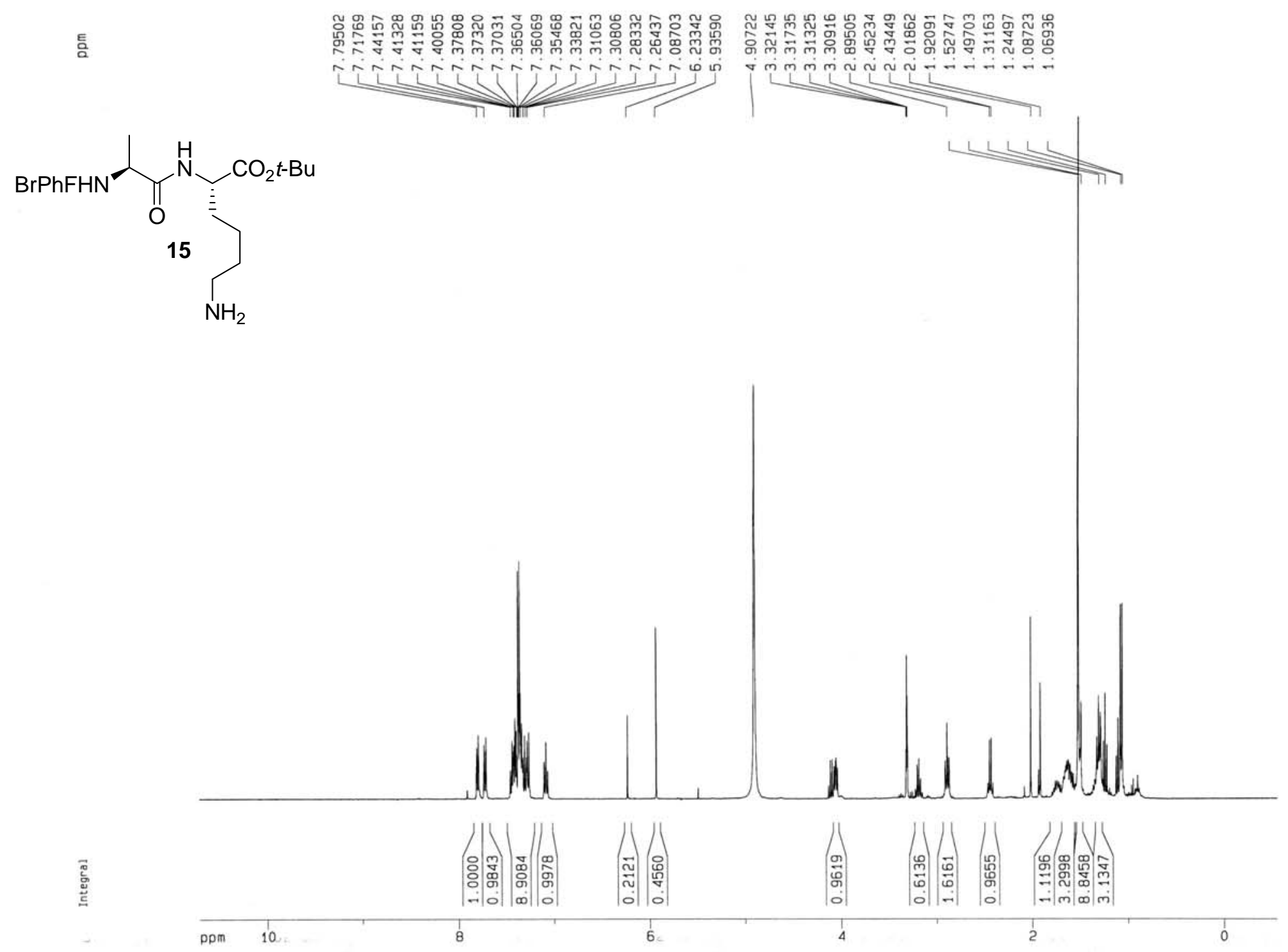




$$
\text { II }
$$




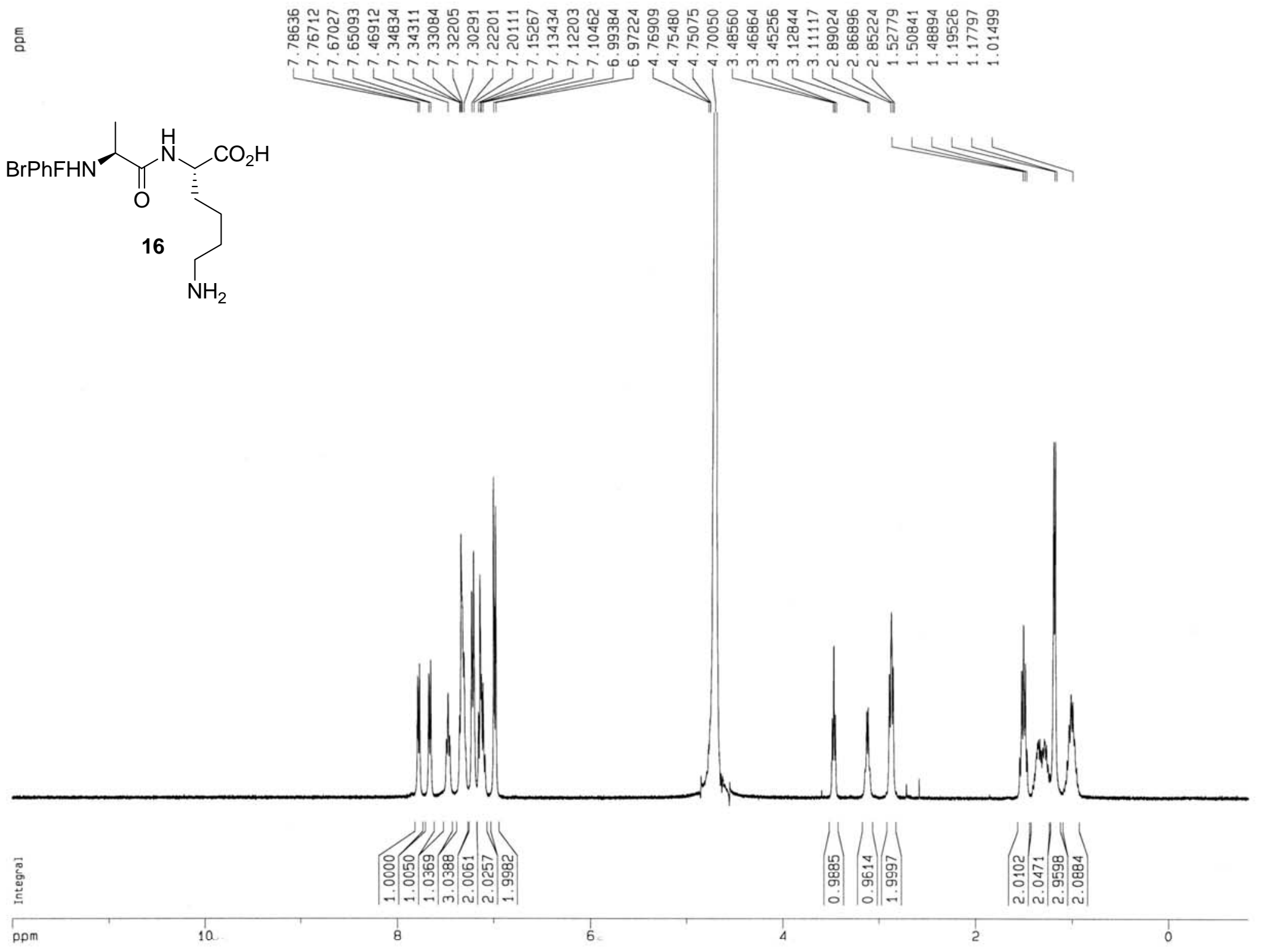



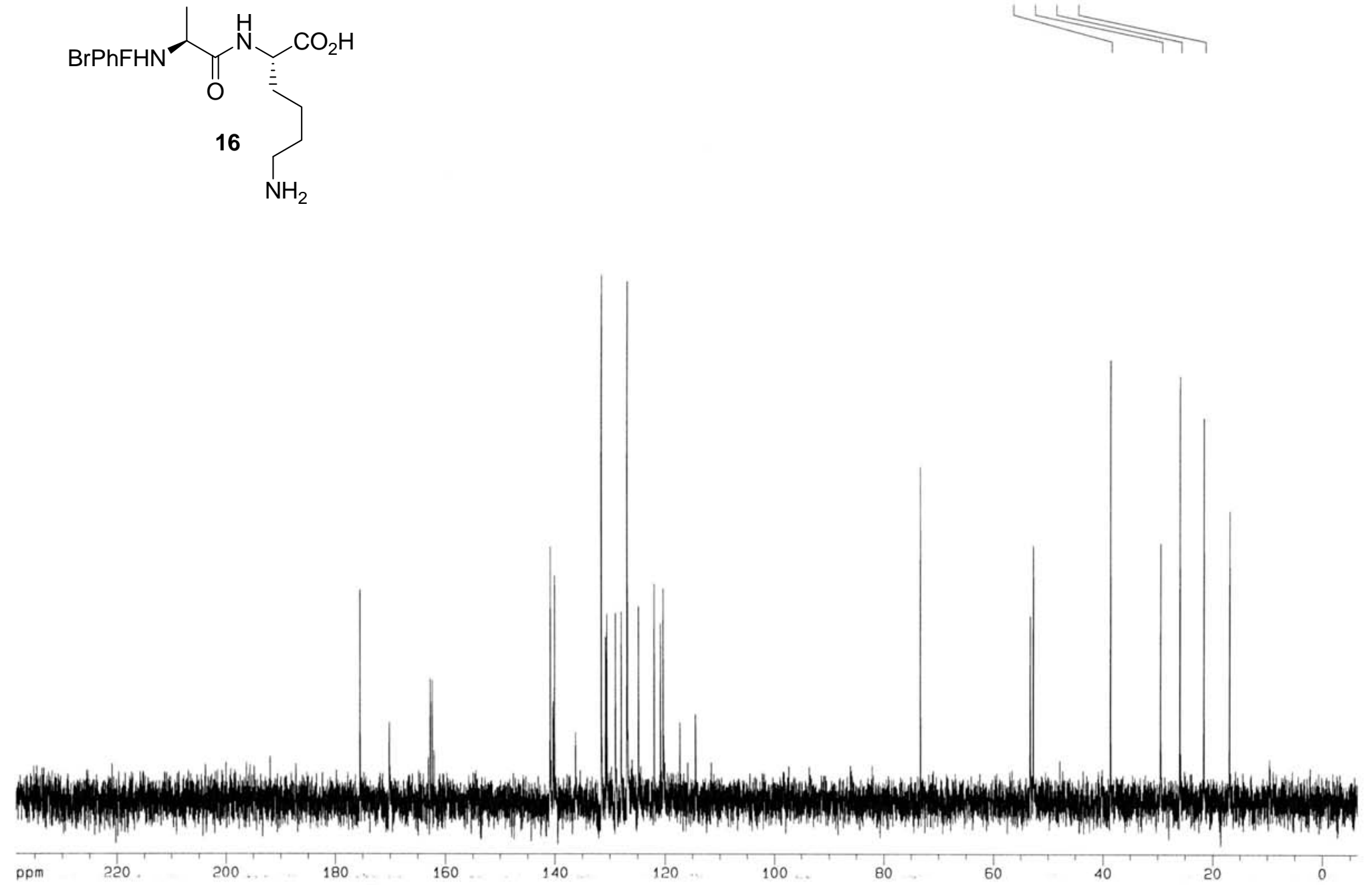


\section{Initial mixture}

Series: 0877

\section{D-7000 HPLC System Manager Report}

Analyzed: 03/10/04 17:00

Reported: 04/10/04 10:32

Data Path: D:\WIN32APP \HSM \samples \DATA \0877\

Processing Method :DAM OBTO100B-40min

Sample Name: Mix

Name: Mix

Chrom Type: Fixed WL Chromatogram, $214 \mathrm{~nm}$

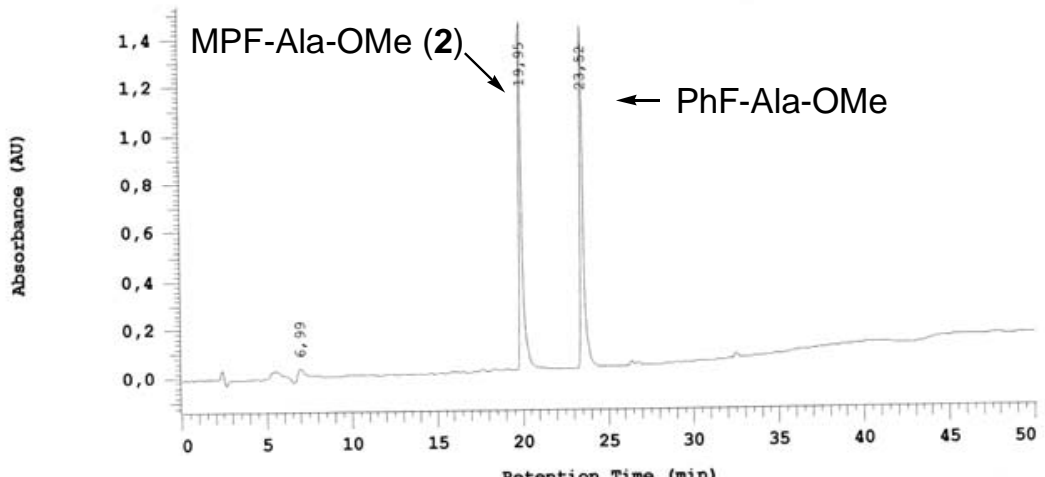

Acquisition Method:

Retention Time (min)

in $40 \mathrm{~min}$ then $10 \mathrm{~min}$ at $1008 \mathrm{~B}$.

(A : H2O 0.18 TFA, B : ACN 0.18 TFA).

Developed by: DAM

Pump A Type: L-7100

olvent A: $\mathrm{H} 200.18$ TFAA

Solvent C:

Solvent B: ACN 0.18 TFAB Solvent D:

Method Description: gradient $0 \mathrm{~B}-100 \mathrm{~B} \_40 \mathrm{~min}$-50min Chrom Type: Fixed WL Chromatogram, $214 \mathrm{~nm}$

\section{After 20 min of solvolysis}

Series: 088

\section{D-7000 HPLC System Manager Report}

Analyzed: 03/10/04 23:41

Reported: $04 / 10 / 04 \quad 10: 26$

Processed: 04/10/04 00:32 Processing Method : DAM OBTO100B-40min

Sample Name: $20 \mathrm{~min}$

Chrom Type: Fixed WL Chromatogram, $214 \mathrm{~nm}$

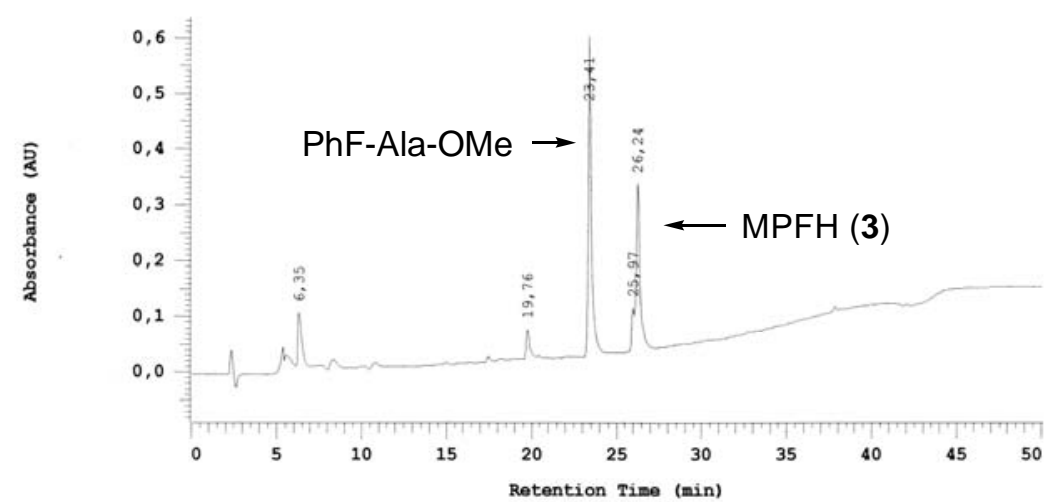

Acquisition Method:

Gradient :

DAM

$08 \mathrm{~B}(100 \% \mathrm{~A})$ to $1008 \mathrm{~B}(08 \mathrm{~A})$ in $40 \mathrm{~min}$ then $10 \mathrm{~min}$ at $1008 \mathrm{~B}$.

(A : $\mathrm{H} 200.18 \mathrm{TFA}, \mathrm{B}: \mathrm{ACN} 0.18 \mathrm{TFA}$ ).

Pump A Type: L-7100

Developed by: DAM

A: $\mathrm{H} 20$ 0.1\% TFAA

Solvent C:

Solvent B: ACN 0.18TFAB

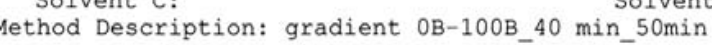

Chrom Type: Fixed WL Chromatogram, $214 \mathrm{~nm}$ 


\section{Initial mixture}

Series: 1028

\section{D-7000 HPLC System Manager Report}

Analyzed: 21/10/04 23:10

Reported: 22/10/04 12:12

Data Path: D: \WIN32APP\HSM \samples\DATA 1028

Processing Method : DAM OBTO100B-40min

Sample Name: mix

Chrom Type: Fixed WL Chromatogram, $214 \mathrm{~nm}$

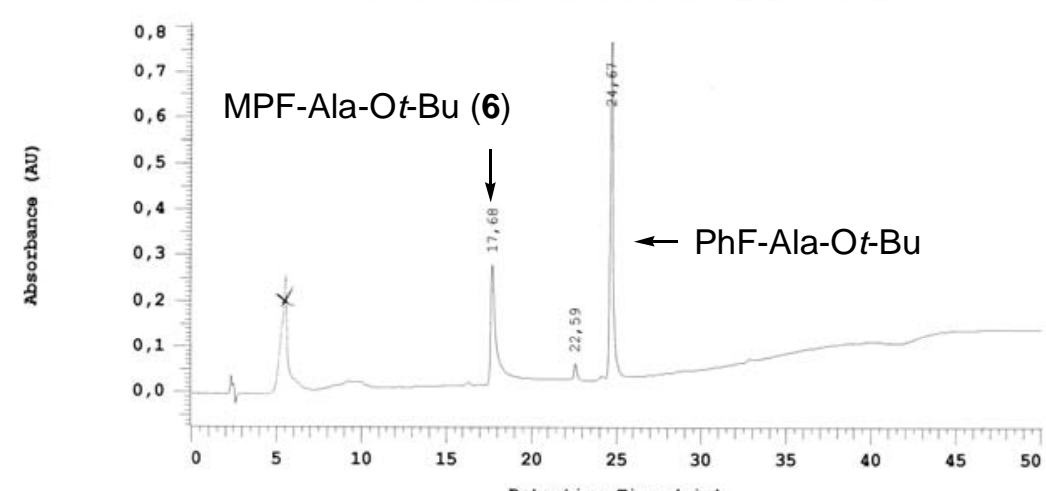

Acquisition Method: Gradient :

Retention Time (min)

Processed: 22/10/04 00:01

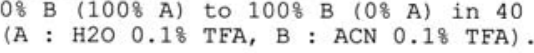

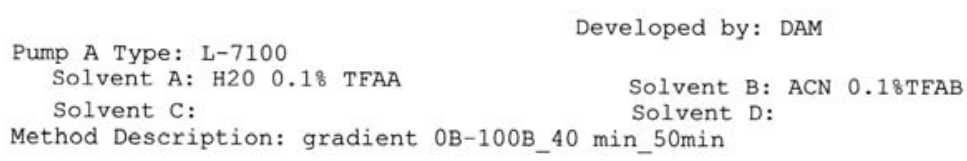

Developed by: DAM

Solvent B: ACN $0.18 \mathrm{TFAB}$

Method Description: gradient $0 \mathrm{~B}-100 \mathrm{~B}_{-} 40 \mathrm{~min}$-50min

Chrom Type: Fixed WL Chromatogram, $214 \mathrm{~nm}$
Series: 1029

\section{D-7000 HPLC System Manager Report}

Analyzed: 22/10/04 00:16

Reported: 22/10/04 12:14

Data Path: D: \WIN32APP \HSM \samples \DATA $\backslash 1029 \backslash$

Processing Method :DAM OBTO100B-40min

Sample Name: $5 \mathrm{~min}$

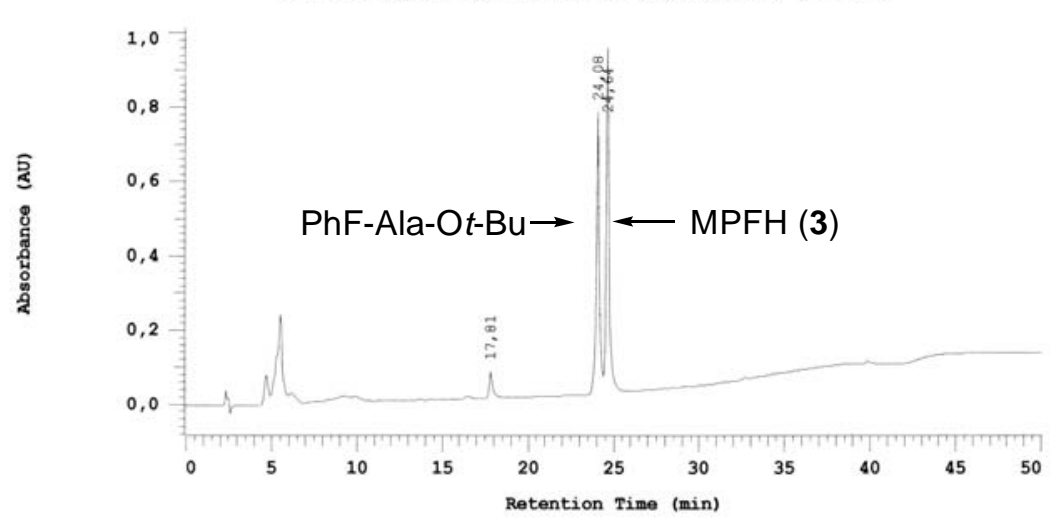

Acquisition Method:

Gradient

$0 \%$ B $(100 \% \mathrm{~A})$ to $100 \% \mathrm{~B}(0 \% \mathrm{~A})$ in $40 \mathrm{~min}$ then $10 \mathrm{~min}$ at $100 \% \mathrm{~B}$.

(A : H2O 0.18 TFA, B : ACN 0.18 TFA).

Pump A Type: L-7100

Developed by: DAM

Solvent A: $H 20 \quad 0.1 \%$ TFA

Solvent C:

Solvent B: ACN 0.18 TFAB Solvent

Method Description: gradient $0 \mathrm{~B}-100 \mathrm{~B} 40 \mathrm{~min} 50 \mathrm{~min}$

Chrom Type: Fixed WL Chromatogram, $214 \mathrm{~nm}$ 